Contemporary Engineering Sciences, Vol. 7, 2014, no. 24, 1321 - 1327

HIKARI Ltd, www.m-hikari.com

http://dx.doi.org/10.12988/ces.2014.49163

\title{
Real-Time DJING + VJING with Interactive
}

\section{Elements}

\author{
Haehyun Jung \\ Graduate School of Advanced Imaging Science \\ Multimedia \& File, Chung-Ang University, Korea
}

Joohun Lee

Dept. of New Media Arts, Dong-Ah Insitute of Media and Arts, Korea

Hyun-Ju Choi

Department of Culture \& Arts Managenment Sangmyung University, Korea

\section{Hyunggi Kim}

Graduate School of Advanced Imaging Science, Multimedia \& File

Chung-Ang University, Korea

(Corresponding Author)

Copyright (C) 2014 Haehyun Jung, Joohun Lee, Hyun-Ju Choi and Hyunggi Kim. This is an open access article distributed under the Creative Commons Attribution License, which permits unrestricted use, distribution, and reproduction in any medium, provided the original work is properly cited.

\begin{abstract}
Development of various media in contemporary society has increased the demand for interactive media and video that uses projection mapping in broadcast, concert, theatre, musicals and so on rather than merely selecting video clips to play, and computer technology development has introduced interactive functions that simultaneously process the audience reaction. While selecting video clips require large $\mathrm{OB}$ vans and equipment of broadcasting equipment switcher, smaller size of DJing and VJing and various types of sensor allowed simultaneous screen and sound from audience motion or performance motion, and based on this, we created DJing and VJing using simultaneous interactive elements.
\end{abstract}


Keywords: Interactive, DJING, VJING, Performance

\section{Introduction}

Our common interest is the technology's development along with interest on art and games.This emerged technology gave a birth to Interactive Media as a new genre art, and it is in fast development phase. Until now the Media Art is considered as one of pure art but they are entering one of the applied areas.[1] Modern society sees newly added value which is created by synergy of art and technology. The integrated technology is named as Culture Technology (CT).[2] For the implementation by DJING + VJIN, while classic Vjing by Visual Jockey was on-going, other performance techniques like improvised, and upto-situation's use of Movie clips or Still Image through projection was performed. [3] As Ditital related industry is developing, performance art is progressing accordingly, and there are increased numbers of works including interactivity as time goes by [4]. Such development of advance technology has increased the role of new communication media, the importance of mutual communication in performance and screen field, and various shows that are interactive and surpassing the limit of screen and shows that is arranged in advance by performing with film content that uses performer's motion and gesture. This study has developed a real-time interactive work with gesture and motion recognition using DJing and VJing from audience participation and performance.

\section{Understaning VJING}

VJING is a 'playing' visual images with music in live performance.[5] Since Motion graphic and VJing can implement design, video, and music at the same time, this should be regarded as different one from existing imaging area.[6] modul8 is a live video mixer program (Fig.[1]). Mapping with keyboard, MIMI mixer is convenient. With the video fixed to virtual clip, user can choose video, change color and control simple elements such as speed. Simple filter application such as blur and noise is also possible. 


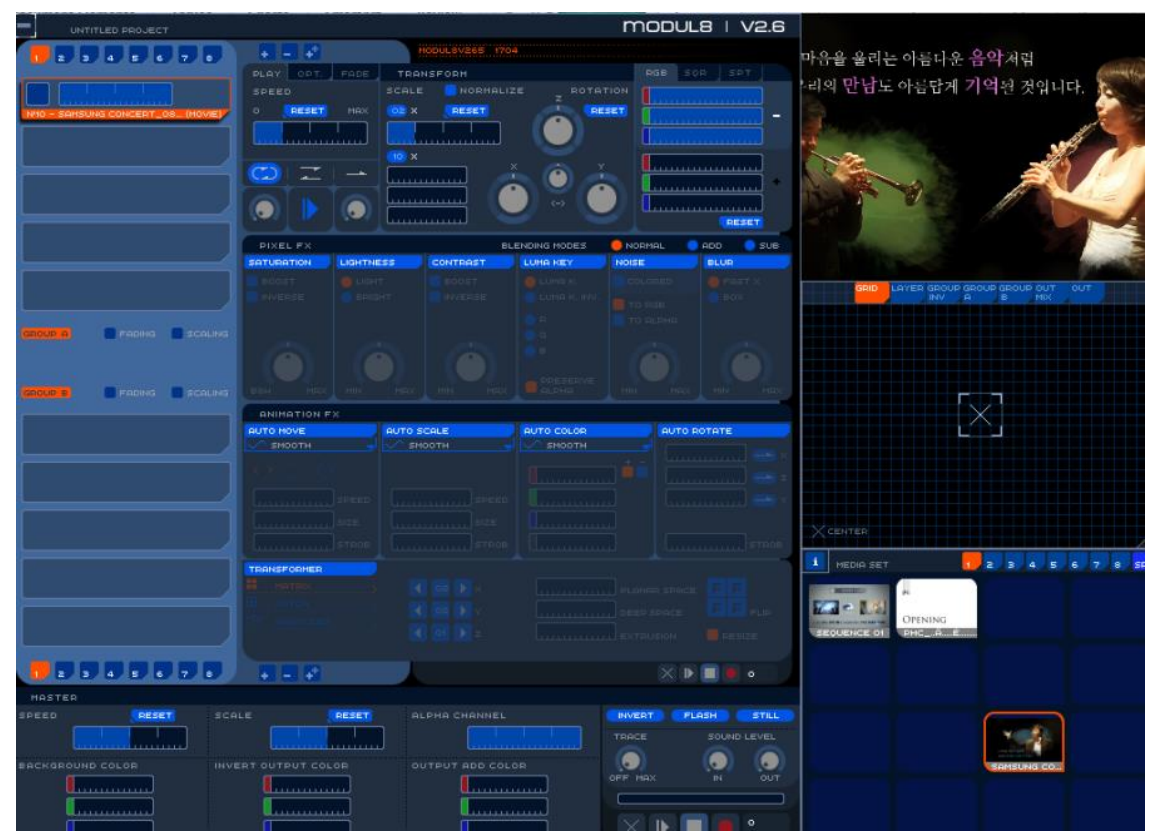

[Fig1] modul8

In the case of Resolume Arena, Effects functions and Adobe After Effects functions on top of modul8 function of [Fig1] has become possible, and using microphone input and sound wave from wav file, user can change screen. MIDI and KEY mapping is possible, and using network communication such as OSC and Syphon, the program is compatible with other programs such as Processing, VVVV, MAX/MSP Jiter and even other programs like MADMAPPER.

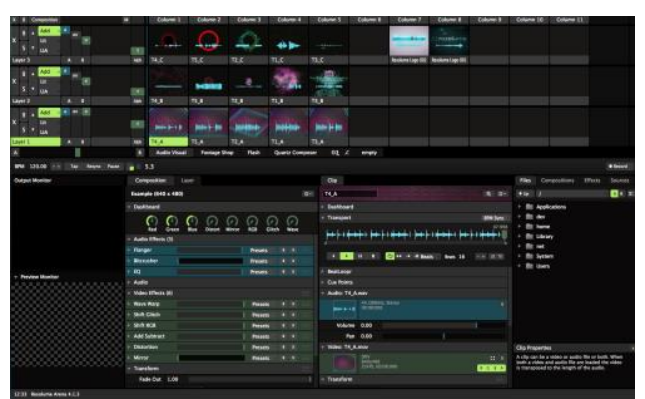

[Fig2] Resolume Arena

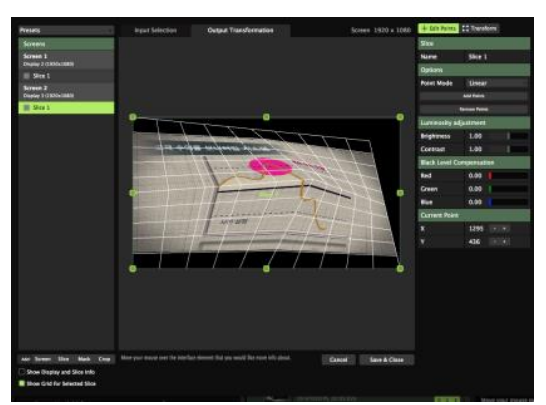

[Fig3] Screen Waping(Arena)

Madmapper performs simple functions such as Opacity, Blend mode, RGB, FLLP, Mesh Warping, MASK of image by using polygon and mask with input of images, movies and syphon. Rather than using various input, simple input can be used with Resolume Arena through Syphon communication. 


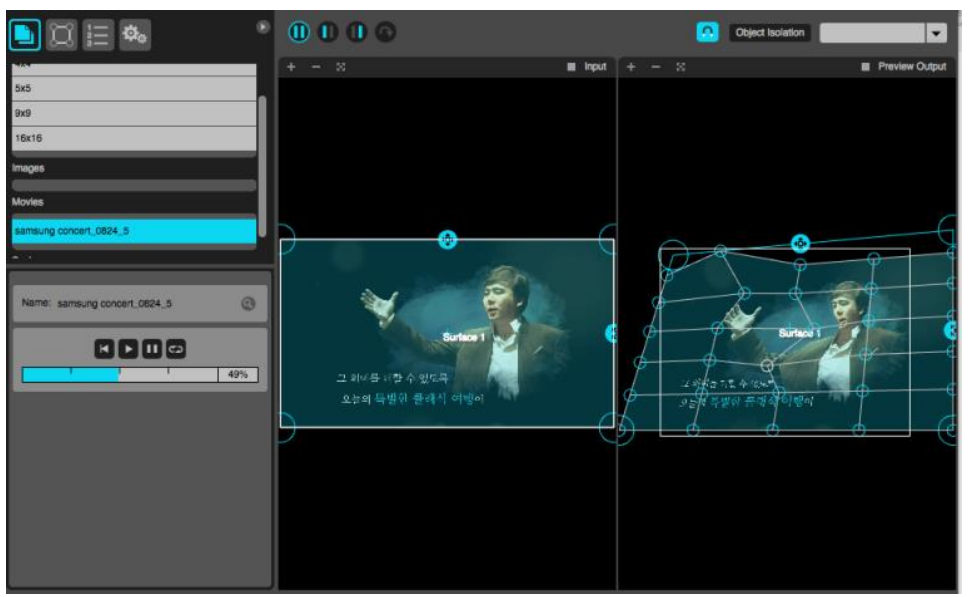

[Fig4] MadMapper

\section{Interactive Media Performance Cases}

Interactive media performance is a new form of show introduced by media development, which creates mutual communication between performer and audience that is not arranged in advance and appeals to various senses at the same time. [7] Installation arts with interactive media art is to create a virtual space based on digital technologies and audiences are allowed to behave as they please to interact with an artwork within that space. These features could stimulate children' $\mathrm{s}$ interest, and these whole processes would make them move their bodies and interact with others.[8] Well-known interactive media performance cases include Scotte Snibbe and Deep Walls who traced audience motion with video camera. This installation art with video camera induces audience participation by reflecting the audience with their shadows. It is a screen based interactive installation performance art that is interactive and through which audience become the work itself in the performance.

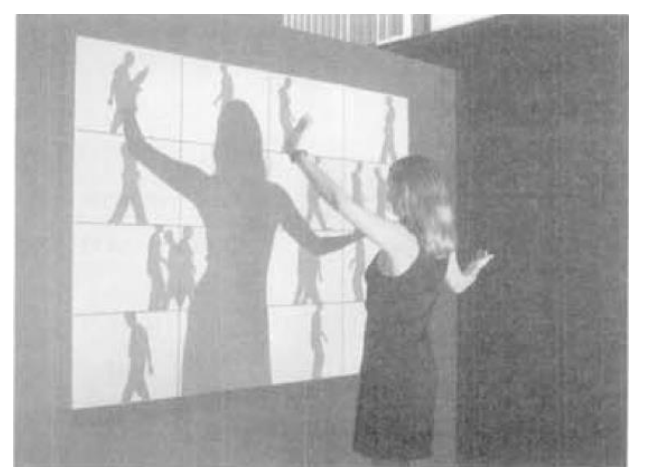

[Fig5] Scotte Snibbe, "Deep Walls” 
Rain, Sponge Bob's one of the commercial exhibitions and a good example of combining audience and screen contents, creates a mutual communication between audience and screen contents with a screen projection and an umbrella, based on audience location recognized by Microsoft's Kinect.

As seen in these two interactive media performance cases, this project also allows anyone to be the performer by their gesture or location recognition to experience DJing and VJing that was conventionally thought as an area of film editing experts, inducing active participation. Such participation is a direct experience that can provide much greater impression and communication.

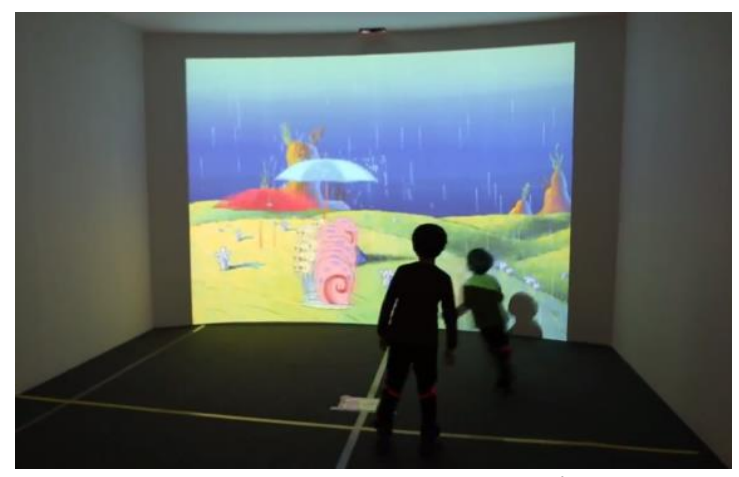

[Fig6] Sponge Bob "Rain”

\section{Application of Real-time Interactive Media Art}

Color Xylophon drops virtual balls with various colors to the floor on a screen that reflects audience like a mirror and makes the balls pop with various sound when they touch the audience. Desired effect: Children will stretch their hands and jump around anticipating the chance to burst the ball of their favorite colors as they keep falling. During the process they get to perceive visual stimulus that is various colors of the ball, an auditory stimulus that is various sounds of xylophone (hearing) and also a stimulus from body movement. Also, seeing oneself reflected in the screen can remind one of looking into the mirror and holds a meaning of self- perception.[9]

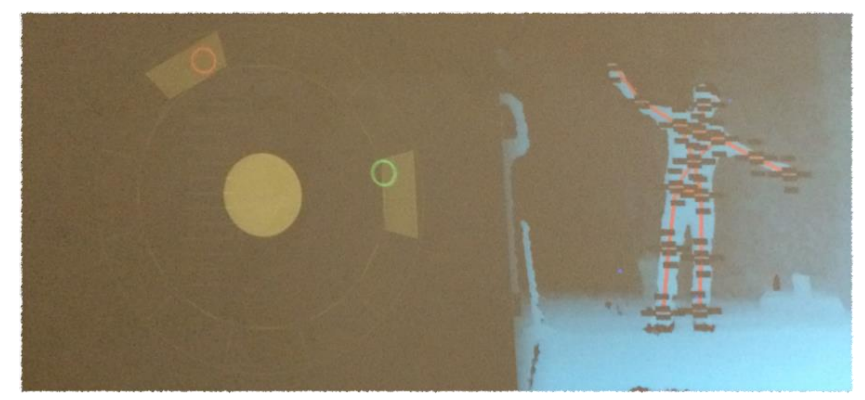

[Fig7] Real-time selection with Kinect 
VJing at 2014 Loop Barcelona exhibition allowed audience to choose and switch videos simultaneously with their gestures. Kinect is a device that provides an experience game and entertainment with user's body instead of controller [10]. With Synapse for Kinect and Processing, Resolume Arena, Kinect can allow simultaneous control over track and source of the screen contents.

\section{Conclusion}

This project was designed to overcome the simple video image choices by screen buttons and to allow simultaneous control over DJing and VJing using media performance and screen control for anyone to participate by incorporating digital media and technology. Interaction is based on simultaneous screen control by audience reaction by using Synapse for Kinect and Processing, Resolume Arena. Processing allows audience to simultaneously control the screen, increase the level of audience immersion and provide a more realistic experience of virtual space. Media performance for real-time interaction reacts directly to the participating audience and extends their experience. [11] Thus, linking audience gesture and control of the virtual space projected in image $(2 \mathrm{D}, 3 \mathrm{D}, 4 \mathrm{D})$ and mapping facial characters by trait-based face recognizing algorithm are also expected to develop in the future. Applying those technologies in media art, interactive media, VJing and other beneficial communication media is anticipated.

Acknowledgements. This research was supported by Chung-Ang University's Cross Functional Team (CFT) Program under Brain Korea 21 PLUS Project in 2014.

\section{References}

[1] Yun-Tae Kim, Applying Interactive Media Art to VJing, The Korea Contents Association, p80, 2007

[2] Kim Jin-Gyu, A Study on the Concept of Culture Technology (CT) and Methods of CT R\&D Projects , Korea Creative Content Agency, pp5, 2013

[3]Kokyuheun, "Real-time interactive pefomances and its distintions from conventional theatre performances", SungKyunKwan Univ, 2002

[4]Yun-Tae Kim, Applying Interactive Media Art to VJing, The Korea Contents Association, p81, 2007

[5] Yun-Tae Kim, Apllying Interactive Media Art to VJing, The Korea Contents Association, p81, 2007 
[6] Haehyun Jung, Real-time Interactive Media Art Implementation with DJING + VJING,

[7] Lee Seunghyun, Study on the Achievment and Limitation of Interactive Media Performances, p25, 2008

[8] Joohun Lee, Interactive Media Artworks as Play Therapy through Five Senes, p21, 2013, Games and Graphics

[9] Joohun Lee, User Interface of Interctive Media Art Works using Five senses as Play Therapy, p141, 2014, International Journal of Bio-Sicence and Bio-Techonology

[10] http://ko.wikipedia.org/wiki/\%ED\%82\%A4\%EB\%84\%A5\%ED\%8A\%B8

[11] Jin Kyung Ahn, Study on narrative, space and the role of audience by participatory transmedia performance: the basis of Cie Mulleras troupe's projects (2012), 34.

\section{Received: August 27, 2014}

